\title{
Effect of reinforcement on tensile behaviour of MWCNT Filled Thermoplastic Composites
}

\author{
Shrinatha R. Katti* \\ Department of Mechanical Engineering, NIE \\ Institute of Technology, Mysuru, Karnataka, \\ India
}

\author{
M. V. Achutha \& B. K. Sridhara \\ Department of Mechanical Engineering, The National \\ Institute of Engineering. Mvsuru. Karnataka. India
}

\author{
D.O.I-10.51201/12316 \\ https://doi.org/10.51201/12316
}

\begin{abstract}
Polypropylene (PP) is one of the most widely used engineering polymers. In this study, multiwalled carbon nanotube (MWCNT) has been melt blended into PP using twin screw extrusion process, indifferent ratios for two different set of composites. For first set, 2.5, 5 and $10 \mathrm{wt}$.\%of MWCNT was used whereas for another set 1.25, 3.75 and 7.5\% were the values. The extruded pellets were then injection moulded using a 50 Ton machine to produce PP+MWCNT composite flattest specimens according to ASTM standards and were used to carry out the tensile tests. While there is a huge reduction in failure strain and a marginal increase in ultimate tensile strength, there was nearly $65 \%$ increase in young's modulus with MWCNT as reinforcement. These experimental values were compared with the theoretical values calculated using Halpin-Tsai model for randomly oriented fibres.There was significant deviationof the theoretical data from the experimental data. The comparison and analysis of theoretical and experimental results are carried out in the present work. A mathematical model equation has been fit for the variation of young's modulus in both the sets of composites. The variation has been found to be constant in terms of the parameters of the equation. This shows the variation of the tensile properties are proportional to the degree of reinforcement.
\end{abstract}

Keywords-Polypropylene, carbon-nanotubes, twin-screw extruder, nano-composites, Halpin-Tsai.

\section{INTRODUCTION}

Owing to their broad range of properties and manufacturability, both synthetic and natural polymers play an essential and useful role in everyday life. Inspite of their attractive properties, they have low toughness due to their tendancy to be string and brittle but still they are highly deformable. This results in low energy absorption capacity during failure. The combination of the existing usage with specific improvement targets for expanded use makes polymers a prime candidate for composite matrices. Existing research also indicates that polymer composites with nanofillers show superior performance versus microfillers [1].

Polypropylenes, polyamide (Nylon) belong to the family of crystalline polymers. Polypropylene (PP) has a wide spectrum of applications including packaging and labeling, textiles (e.g., ropes, thermal underwear and carpets), stationery, plastic parts and reusable containers of various types, laboratory equipment, loudspeakers, automotive components, and polymer banknotes due to its processability, good balance of physical properties and price. The structure of PP is chemically simple. This makes it good choice as matrix material in understanding the properties of composites.

A Carbon Nano Tube (CNT) is an allotrope of carbon with a cylindrical form and subdivided into singlewalled (SWCNT) and multi-walled CNTs (MWCNTs). They are formed by rolling the one-atom-thick grapheme sheet into cylinders at a specified angle, variation in which will lead to different CNT structures. CNTs have $\mathrm{sp}^{2}$ bond between carbon atoms which is responsible for its high tensile strength and elasticity modulus [5]. MWCNT will have multiples layers of graphene sheets (concentric cylinders) which make them incredibly strong fibers. SWCNT are well suited for electrical and thermal conduction. The strength of a MWNT is ten times higher than any other known fiber. 
Carbon nanotubes (CNTs) are of particular interest as a reinforcement option for these composites due to their remarkable mechanical properties. CNTs are shown actually and theoretically to have tensile strengths up to $60 \mathrm{GPa}$, substantially stronger than steel with approximately one sixth the density. Their special mechanical properties are due to strong bonding between the carbon atoms that form carbine plane. The bond length is $0.142 \mathrm{~nm}$ which is lower than that in diamond $(0.154 \mathrm{~nm})$.Tensile strength of CNT is high, which is 75 times than that of steel filaments of the same size, also 15 times higher than that of carbon fibers. On the other hand, the densities of CNT are as low as $1.3 \mathrm{~g} / \mathrm{cm}^{3}$, which is one sixth of that of steel. CNTs are suitable as reinforcement in composites used as field effect transistors, nanowires, fuel cells and sensors, due to the outstanding physical properties such as low specific gravity, mechanical strength, thermal stability, and electrical and thermal conductivity [2]

The structure of PP is chemically simple and hence the effect of adding MWCNT to PP can be evaluated. Carbon nanotubes (CNTs) are promising additives for polymer composites due to their special excellent mechanical, electrical and thermal characteristics. PP/CNT composites produced at laboratory scale showed that low electrical percolation threshold and good reinforcement characteristics may be achieved [1]. CNT/polymer composites combine the good processability of the polymers with the excellent mechanical and other functional properties of the CNTs [4]. PP / CNT would produce very strong materials which are light at the same time. Plastics enhanced with CNTs might be a new family of light and strong composites [3].

With this background MWCNT has been selected as the reinforcement with the Polypropylene matrix. The tensile properties for the composite are compared with that of the natural polymer. These results have been analysed and the results are further used to conduct tests under cyclic loading.

A great deal of theoretical work has been carried out since the 1950s with the aim of modelling the mechanical properties of fibre reinforced composites. While some of these models are quite sophisticated, two of the simplest and most common models are the rule of mixtures and the Halpin-Tsai equations. Halpin-Tsai model was originally developed for continuous fibre composites and follows on from the work of Hill. These works are reviewed extensively by Coleman et. al [6]. This model presents the equations connecting the 1/D ratio of various fibres and their young's moduli with that of matrix material to calculate the Young's modulus of the new composite material. This model also considers different conditions of fibre dispersion. For the present study, Halpin-Tsai equation for randomly oriented fibres is considered and is given below.

$$
\begin{gathered}
\frac{Y_{c}}{Y_{m}}=\frac{3}{8}\left[\frac{1+\zeta \eta_{L} V_{f}}{1-\eta_{L} V_{f}}\right]+\frac{5}{8}\left[\frac{1+2 \eta_{T} V_{f}}{1-\eta_{T} V_{f}}\right] \\
\text { where } \eta_{L}=\frac{\frac{Y_{f}}{Y_{m}}-1}{\frac{Y_{f}}{Y_{m}}+\zeta}, \quad \eta_{T}=\frac{\frac{Y_{f}}{Y_{m}}-1}{\frac{Y_{f}}{Y_{m}}+2} \text { and } \zeta=\frac{2 l}{d}
\end{gathered}
$$

where $\mathrm{Y}_{\mathrm{f}}$ is the fibre modulus, $\mathrm{Y}_{\mathrm{m}}$ is the matrix modulus and $\mathrm{V}_{\mathrm{f}}$ is the fibre volume fraction.

\section{EXPERIMENTAL WORK}

\section{A. Materials}

Polypropylene (PP) is used as the matrix material which is reinforced with the MWCNT. PP used is H110MA grade from Reliance polymers, with Specific gravity of 0.9, MFI 11 and tensile strength 36 $\mathrm{MPa}$ and flexural modulus $1650 \mathrm{MPa}$. Multiwalled Carbon Nanotube was taken from Arkema Inc. (France) with outer diameter of 10-15 nm.

\section{B. Preparation of Composites}


The natural PP granules were mixed with weight percentages of CNT by manual mixing to get the uniform mixing of the materials. Then mixture was passed through the twin screw extruder for getting granules in perfect blend of PP \& CNT. Compounding was done with the main extruder rotating at 210 $\mathrm{rpm}$ and the feeder at 20-30 rpm. The temperature profile along the barrel and die with 7 zones was set to $172 / 187 / 195 / 191 / 204 / 220 / 207$ and the melting temperature at $167^{\circ} \mathrm{C}$ for all the experiments.

The composite blends containing different compositions of the reinforcement material, MWCNT are fed into the Injection molding machine of 50 ton capacity to mold them into tensile and flexural test specimens according to ASTM standards (ASTM D638 - Standard test method for Tensile Properties of plastics.

The $\mathrm{PP}+\mathrm{CNT}$ composites were prepared in two sets of compositions, containing CNT in weight percentages as detailed below.

Table - 1: Composition details of PP + MWCNT composites prepared

\begin{tabular}{|c|c|c|c|c|c|c|c|}
\hline $\begin{array}{c}\text { Matrix } \\
\text { PP }\end{array}$ & \multicolumn{6}{|c|}{ MWCNT Percentage by weight } \\
\hline Set -1 & 0 & & 2.5 & & 5 & & 10 \\
\hline Set -2 & 0 & 1.25 & & 3.75 & & 7.5 & \\
\hline
\end{tabular}

The compositions under Set -2 are selected so as to fall exactly in between those selected under set -1 . These compositional details of the composites are summarized in the Table -1 .

\section{Tensile tests}

The tensile properties of the standard dumbbell-shaped specimens were determined using the standard test method as per ASTM D638. The dimensions like length, width, gage length, radius of the fillet of the test specimens are conforming to the Type- 1 in the standard. The specimen was loaded at a strain rate of 5 $\mathrm{mm} / \mathrm{min}$ which is specified for type-1 specimen. The load and extension at the yield point and the rupture point are noted down. Two specimens are tested under each composition and the average behaviour of both are discussed here.

\section{RESULTS AND DISCUSSION}

Experimental results are analyzed by taking the mechanical properties of Polypropylene as reference. The raw data of the tensile tests are taken and sorted to study the relevant important data. strain and stress values are calculated using the initial length and cross-sectional area values respectively. The overlapping of the stress-strain curves for each composition of reinforcements resulted in the graphs shown in Fig. 1 and Fig. 2. The Young's Modulus is calculated for every composition of CNT. The variation of this modulus for varying reinforcement percentages are depicted by the graph shown in Fig. 3. 


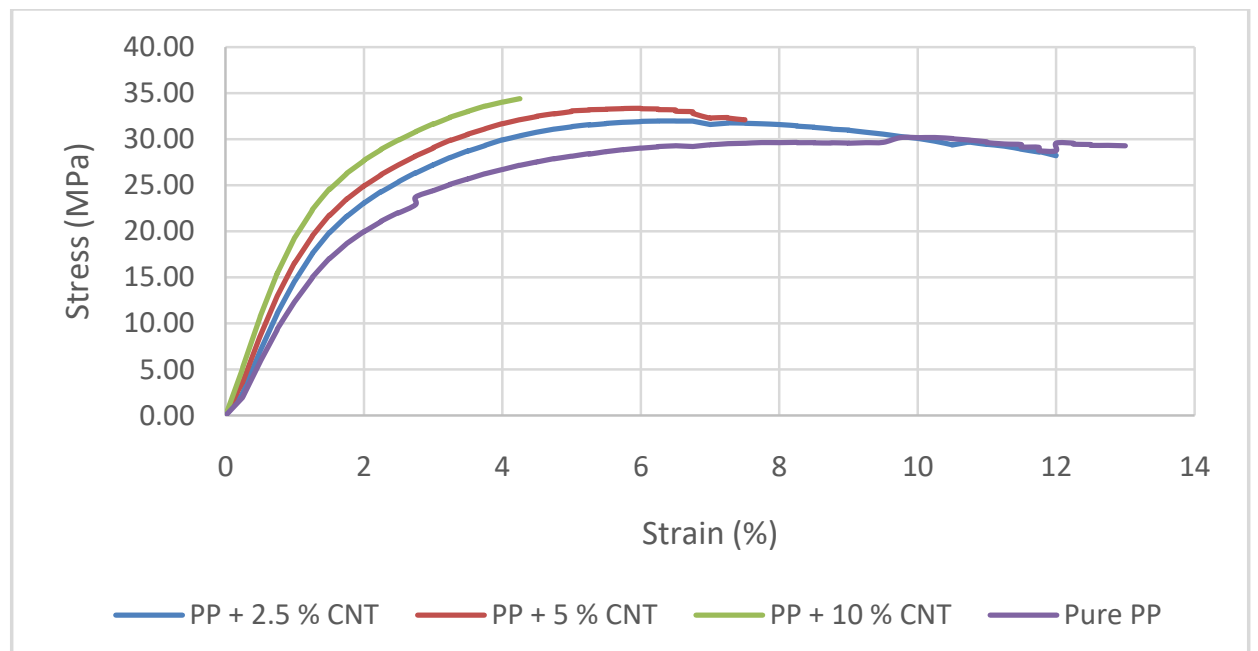

Fig. 1 Stress-Strain plots for various compositions of PP-MWCNT Composites under set -1

Figure 1 shows the effect of adding carbon nanotubes into polypropylene matrix. The failure strain reduces significantly from $12 \%$ to $4.3 \%$, that is nearly $64 \%$ drop in failure strain with the addition of nearly $10 \%$ MWCNT. The ultimate tensile strength increases marginally from $31.7 \mathrm{MPa}$ to $34.4 \mathrm{MPa}$ with the addition of MWCNT.

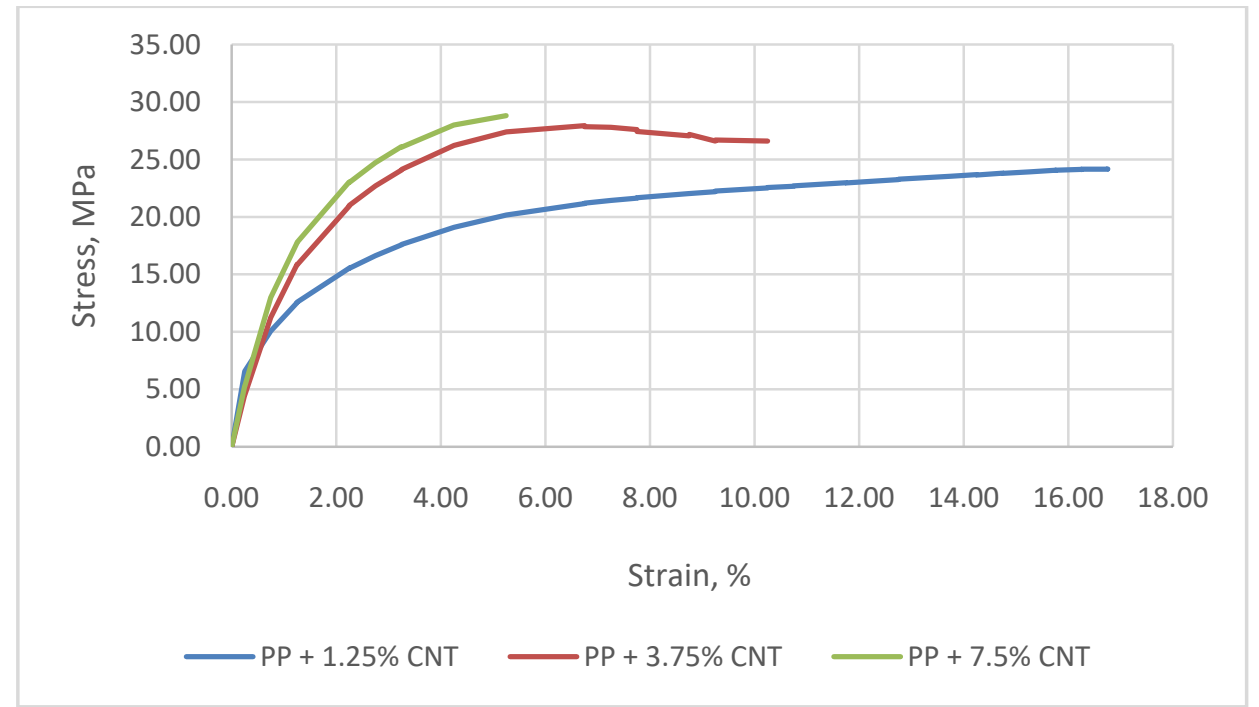

Fig. 2 Stress-Strain plots for various compositions of PP-MWCNT Composites under set - 2

The young's modulus of pure PP is $1.4 \mathrm{GPa}$. When MWCNT is used, there is rapid increase in the Young's modulus by nearly $65 \%$. It has increased from $1.4 \mathrm{GPa}$ for pure PP to $2.3 \mathrm{GPa}$ for $10 \% \mathrm{MWCNT}$ composite. While the set -2 is considered, the Young's modulus has increased to 2.08GPa for the maximum composition of $7.5 \%$. This major effect of reinforcement can be attributed to astonishingly high $\mathrm{E}$ value for MWCNT which acts as stiffer material in the matrix than any other form of carbon.

This trend-line of increase in $\mathrm{E}$ value for both the cases are comparatively depicted in Figure 3 . The variation in the behaviour of composite under the two sets can be clearly seen to be in compliance with each other. The equation for the trend-lines of the curves can be observed. Slope of the curve is 9.026 for 
the set -1 while it is 8.94 for set -2 . This shows set -2 has marginally lower slope value then the other. Also the intercept value is almost equal to 1.4 which the YM value of pure PP.

The experimental results clearly indicate dispersion of MWCNT in PP matrix is a challenge. Addition of CNT definitely increases the modulus values of the composite, while significantly reducing the failure strain. The reason for reduction in failure strain is that MWCNT is high stiffness materials and the interface between these reinforcements and the matrix is weak. During the process of loading the crack gets initiated at the interface and readily propagates across the specimen resulting in fracture of the specimen. One possible way of enhancing the fiber-matrix interface properties is to treat CNT surface with a monomer coating of PP before dispersing them in PP matrix.

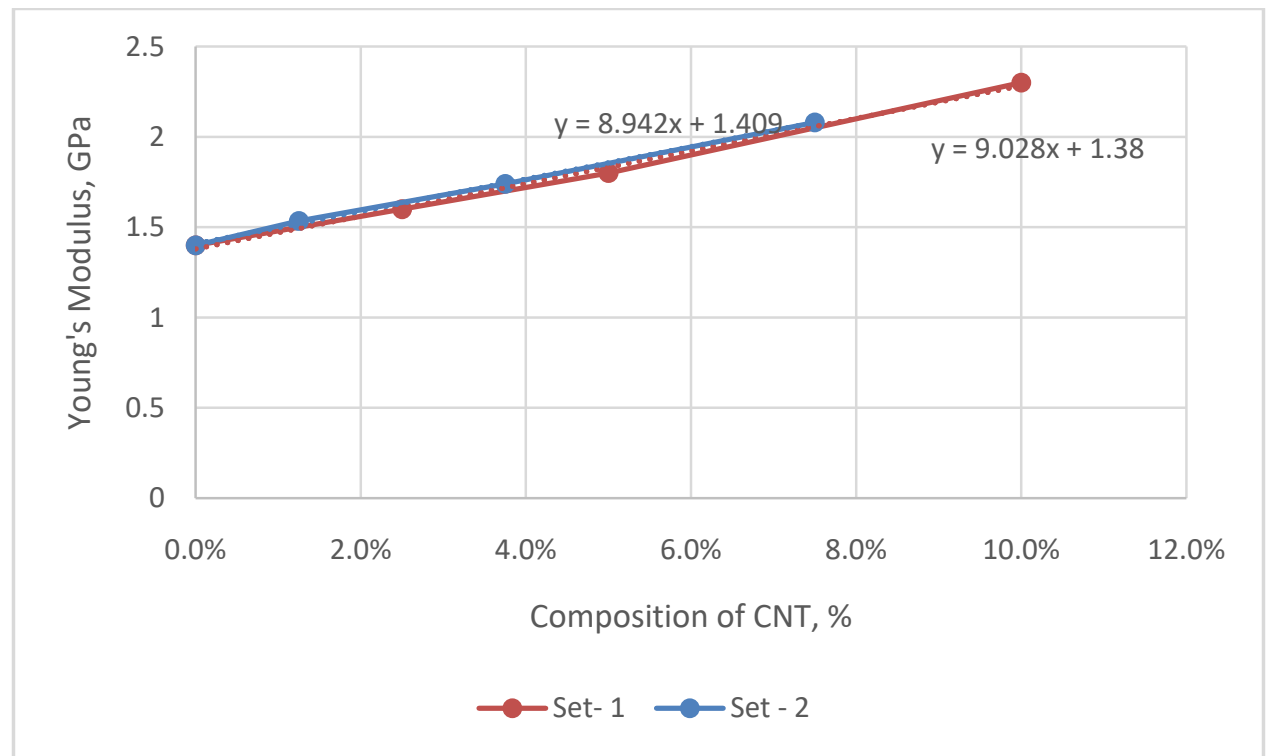

Fig. 3Comparison of variation of Experimental values of Young's Modulus for the two composition sets

\section{Theoretical Modelling}

Using the Halpin-Tsai relations explained in section 1, the young's modulus of the composite with varying compositions of $\mathrm{CNT}$ and $\mathrm{CNP}$ are calculated. These are the values taken for calculations. $\mathrm{Y}_{\mathrm{m}}=$ $1.4 \mathrm{GPa}, \mathrm{Y}_{\mathrm{f}}=1250 \mathrm{GPa}(\mathrm{MWCNT})$ and $\mathrm{Y}_{\mathrm{f}}=12.5 \mathrm{GPa}(\mathrm{CNP})$. The length of the MWCNT is taken to be $20 \mathrm{~nm}$ and length to be $1 \mu \mathrm{m}$ resulting in the $1 / \mathrm{d}$ ratio to be 50 for MWCNT whereas for CNP $1 / \mathrm{d}$ is considered to be 1 .

Figure -6 shows the comparison of the theoretical values for the two sets of compositions of PP _ CNT compositions. They are precisely in correlation woth each other as the values of various theoretical parameters used in the Halpin Tsai equation is maintained constant for the same reinforcement. Just the volume fraction values are changing for the two sets. As the percentage composition selected are exactly in the middle of the values of the other set, the variation trendline have shown great compliance with each other. 


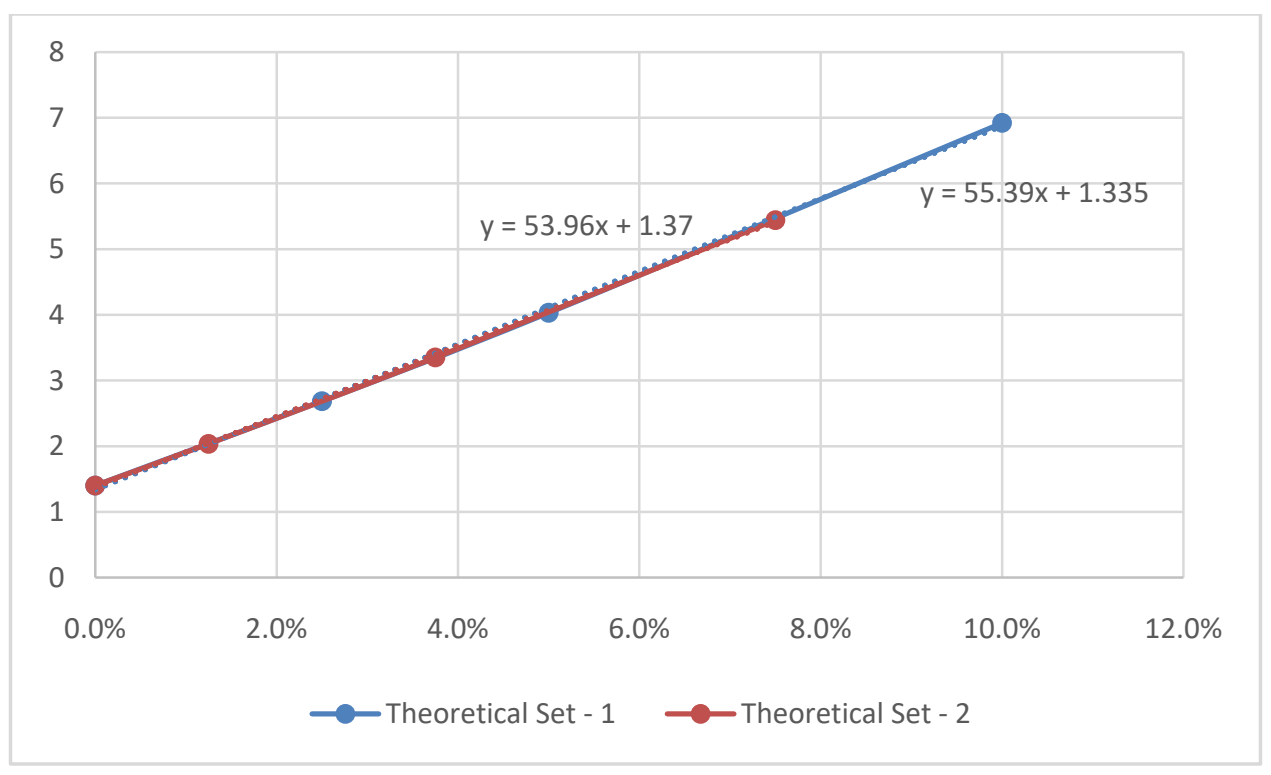

Fig. 4Comparison of variation of Theoretical values of Young's Modulus for the two composition sets

According to the calculations, $\mathrm{Y}_{\mathrm{c}}$ has increased from $1.4 \mathrm{Gpa}$ for pure PP to $6.92 \mathrm{GPa}$ for $10 \% \mathrm{MWCNT}$ which is huge increase. Figure 4 shows the comparison of the experimental and theoretical values of young's modulus for set -1 (upto 10\%). The values are increasing rapidly when MWCNT percentages are increasing The PP + CNT composite has close values in the required range, but the deviation is noticeable. Figure 5 shows the same comparison for set -2 which deviating on the same rate as that for set -1 .

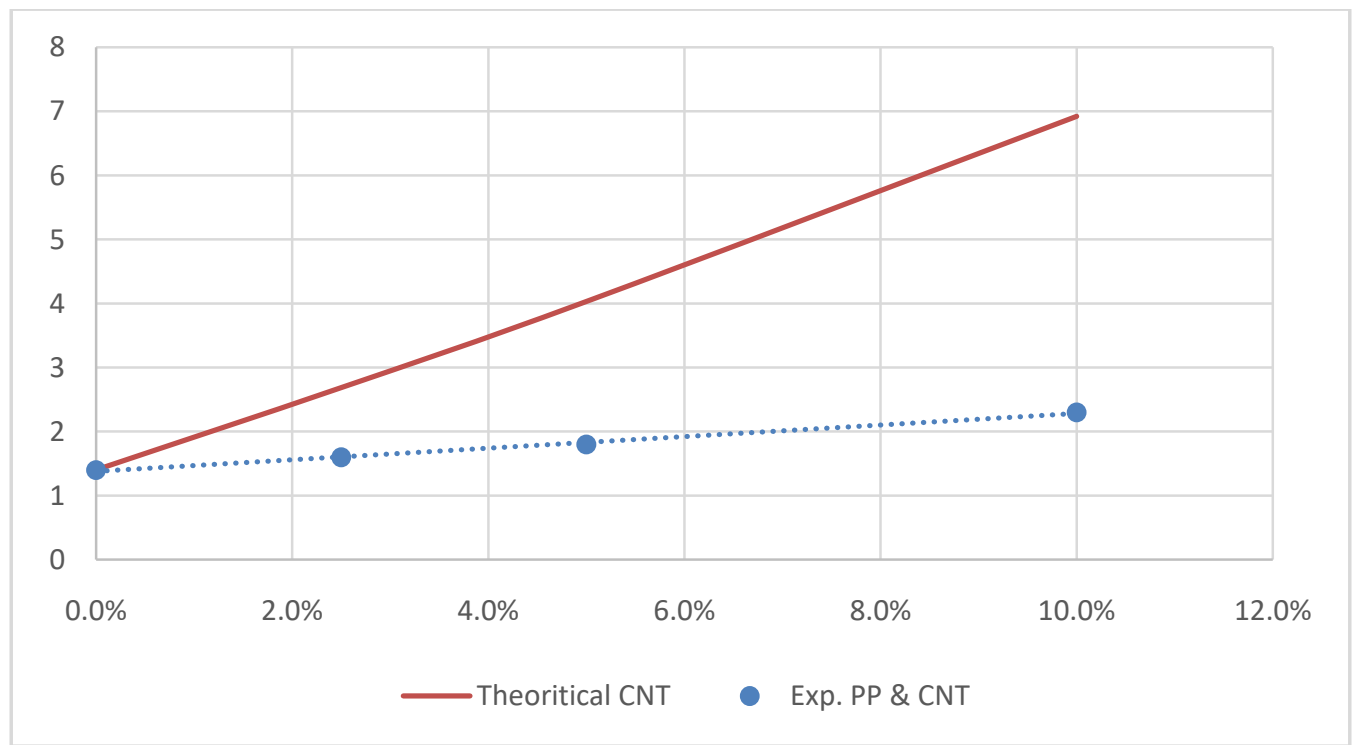

Fig. 5 Comparison of Experimental and Theoretical variation of Young's Modulus for Set - 1

The theoretical and experimental data in case of $\mathrm{PP}+\mathrm{CNT}$ there was significant deviations as evidenced in Figure 4. The Young's modulus for $10 \%$ MWCNT is $2.3 \mathrm{GPa}$ experimentally but is $6.92 \mathrm{GPa}$ theoretically While computing the theoretical values the assumptions that are made is that the modulus of 
CNT is $1.3 \mathrm{TPa}$, which is the highest value reported by the manufacturer in datasheet.However, based on the number of walls in MWCNT, the diameter and length of CNT the value of E can reduce drastically.

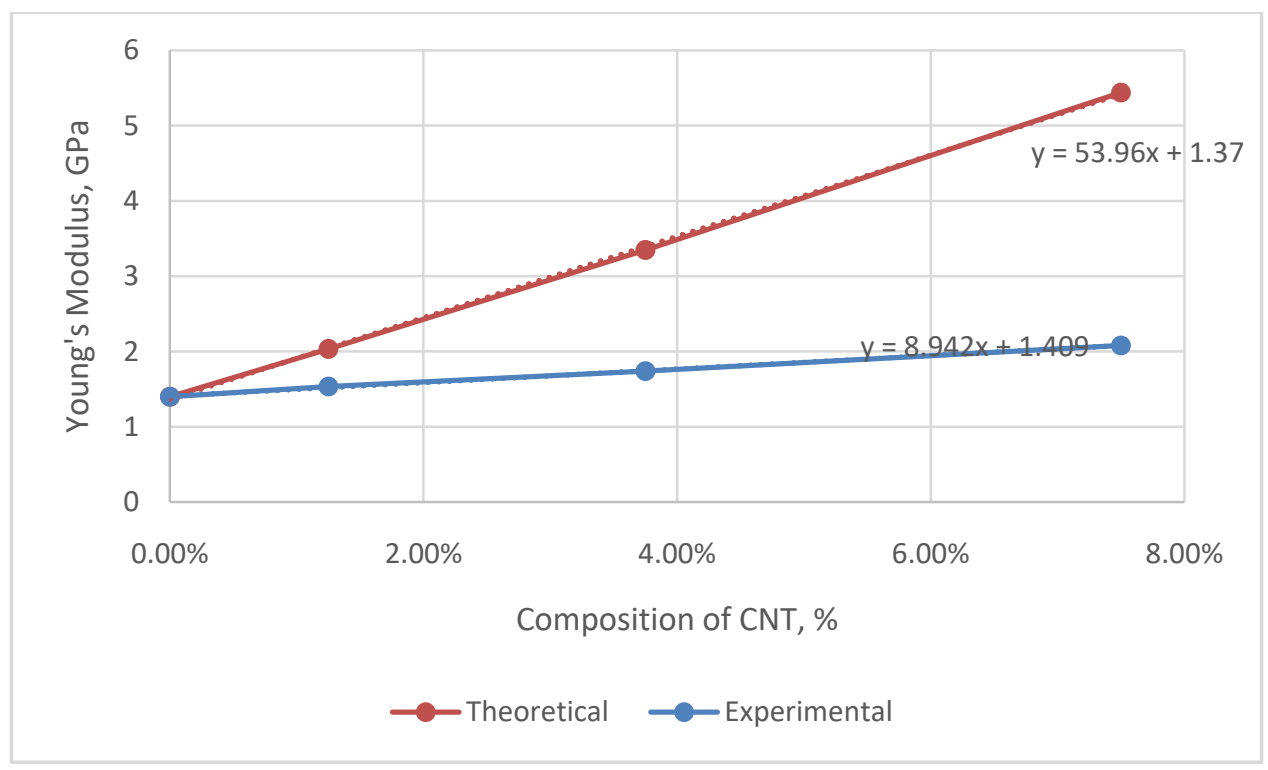

Fig. 6 Comparison of Experimental and Theoretical variation of Young's Modulus for Set - 2

Secondly during the process of mixing MWCNT into PP matrix, the MWCNT may form bundles due to Van-der-waals forces. MWCNT will not be able to stretch itself enabling itself to exhibit its mechanical properties to the full capacity. The net effect could be a reduction in E value of CNT to as low as $0.2 \mathrm{TPa}$. Hence, the Young's modulus taken for calculations in the theoretical models are not met in the practical conditions. This is the reason why the experimental graph is lagging behind than the theoretical line, as shown in Fig. 6.

\section{CONCLUSIONS}

PP / MWCNTcomposites was produced using twin screw extruder and injection molding. These compsites individually contained $2.5,5$ and $10 \%$ by weight of MWCNT in case of Set -1 while it was $1.25,3.75 \& 7.5 \%$ in case of Set -2 . It was observed that the tensile properties of the composite increased drastically, up to $50 \%$, by increasing the reinforcement percentages. MWCNT is observed to influence the composite properties rapidly high due to the special properties of CNT. These experimental values were compared with the theoretical values calculated using Halpin-Tsai model for randomly oriented fibres. When MWCNT is dispersed, the theoretical values were observed to be far more than the experimental results owing to the variation in structure of MWCNT as a reinforcement in polymer matrix. Also the behaviour of the two sets of PP+MWCNT composites showed very good correlation with each other. The equations of the trendlines in both the sets ascertained this tendancy. The trendlines based on theoretical values also almost overlapped showing good compliance. This can be attributed to the dispersion of the MWCNT using the same experimental facilities. Hence we can conclude that the effect of composition percentages in different sets of experiments will not vary the behaviour of the composite.

\section{ACKNOWLEDGEMENT}

The management and research scholars of NIE and NIEIT have supported in bringing out this paper. The authors thank them for their encouragement and collaborative work. 


\section{REFERENCES}

[1] W. Leininger, X. Wang, X. W. Tangpong, M. McNea, Journal of Engineering Materials and Technology, 134, 01, (2012).

[2] R.M. Novais, F. Simon, M. C. Paiva, J. A. Covas, Composites: Part A, 43, 2189, (2012).

[3] A. Szentes, G. Horvath, Cs. Varga, Hungarian Journal of Industrial Chemistry, 38, 67, (2010).

[4] K. Prashantha, J. Soulestin, M. F. Lacrampe, M. Claes, G. Dupin, P. Krawezak, eXPRESS Polymer Letters, 2, 735, (2008).

| [5] Abu Bakar Sulong, Mohd. IkramRamli, Seow Liang Hau, JaafarSaAAhari, Norhamidi Muhammad, Hendra Suherman, Composites: Part B, 50, 54, (2013).

[6] Jonathan N. Coleman, Umar Khan, Werner J. Blau, Yurii K. Gun'ko, Small but strong: A review of the mechanical properties of carbon nanotube-polymer composites, Carbon (Elsevier),44, 1624-1652, (2006) 